\title{
Entanglement between qubits induced by a common environment with a gap
}

\author{
Sangchul Ol* and Jaewan Kin母 \\ School of Computational Sciences, Korea Institute for Advanced Study, Seoul 130-722, Korea
}

(Dated: July 17, 2018)

\begin{abstract}
We study a system of two qubits interacting with a common environment, described by a twospin boson model. We demonstrate two competing roles of the environment: inducing entanglement between the two qubits and making them decoherent. For the environment of a single harmonic oscillator, if its frequency is commensurate with the induced two-qubit coupling strength, the two qubits could be maximally entangled and the environment could be separable. In the case of the environment of a bosonic bath, the gap of its spectral density function is essential to generate entanglement between two qubits at equilibrium and for it to be used as a quantum data bus.
\end{abstract}

PACS numbers: 03.67.Mn, 03.67.Pp, 03.65.Ud, 03.65.Yz

Entangled states, showing nonlocal quantum correlations between subsystems, are indispensable in quantum information processing [1]. A two-qubit interaction is needed to implement two-qubit gates and to make entangled states. In general, there are two types of twoqubit couplings: direct coupling such as dipole coupling in NMR or Coulomb coupling in superconducting charge qubits, and indirect coupling mediated by another quantum system like a harmonic oscillator 2], a cavity mode [3], or even an electron gas [4]. A mediating system is expected to be separable from the qubits, so that its dynamics can be ignored. Recently, Cubitt et al. showed two qubits could be entangled by continuous interaction with a mediating particle in a separable state [5].

Entanglement of qubits and an environment causes the decoherence of qubits, one of the biggest obstacles in quantum information processing. It was believed that qubits should be isolated from the environment. However, it is shown that two qubits without a direct coupling but interacting with the environment could be entangled [6] and even in the steady state [7]. Once qubits become entangled in the steady state, they will never be disentangled due to the irreversible process described in Ref. 7]. This entangling process can not be used for the two-qubit coupling scheme to implement two-qubit gates.

Like a single mediating particle, could an environment comprised of many particles induce the two-qubit coupling? Could it work as a quantum data bus to implement two-qubit gates at equilibrium? In this paper, we address this problem by studying a two-spin boson model shown in Fig. 11. The spin-boson model has been extensively studied to describe dissipative two-state systems [8, 9]. Here we consider two types of the environment: a single mode case and a multi mode case.

Single mode case. - Consider a system of two qubits interacting with a single harmonic oscillator with fre-

\footnotetext{
*Electronic address: scoh@kias.re.kr

${ }^{\dagger}$ Electronic address: jaewan@kias.re.kr
}

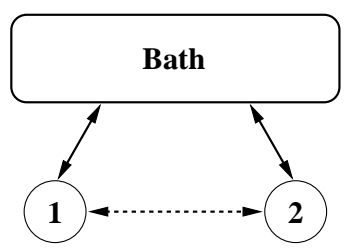

FIG. 1: Two circles refer to two qubits and an oval is an environment. The coupling between qubits and the common environment (solid arrow) induces the indirect interaction between two qubits (dashed arrow).

quency $\omega$, described by the Hamiltonian [5]

$$
H=\hbar \omega a^{\dagger} a+\hbar \lambda\left(\sigma_{1 z}+\sigma_{2 z}\right)\left(a^{\dagger}+a\right),
$$

where $a^{\dagger}$ is a creation operator of the harmonic oscillator, $\sigma_{i z}$ are the $z$-component of the Pauli spin matrices of the $i$-th qubit, and $\lambda$ the coupling constant. The Hamiltonian is solved through the canonical transformation $e^{X}=\exp \left[\frac{\lambda}{\omega}\left(\sigma_{1 z}+\sigma_{2 z}\right)\left(a^{\dagger}-a\right)\right]$ 8, 10]. The transformed Hamiltonian reads

$$
\widetilde{H}=e^{X} H e^{-X}=\hbar \omega a^{\dagger} a-I_{\text {eff }} \sigma_{1 z} \sigma_{2 z}-I_{\text {eff }},
$$

where $I_{\text {eff }} \equiv 2 \hbar \lambda^{2} / \omega$ is the indirect two qubit coupling induced by the harmonic oscillator. A total state is also transformed as $|\widetilde{\Psi}\rangle=e^{X}|\Psi\rangle$.

The time evolution of the total state is given by $|\Psi(t)\rangle=e^{-X} e^{-i \widetilde{H} t / \hbar} e^{X}|\Psi(0)\rangle$. Let us suppose an initial state is given by a product state $|\Psi(0)\rangle=|\psi(0)\rangle \otimes|0\rangle_{E}$ where $|\psi(0)\rangle=a|00\rangle+b|01\rangle+c|10\rangle+d|11\rangle$ is the initial state of the two qubits and $|0\rangle_{E}$ the ground state of the harmonic oscillator. Then the total state at time $t$ is given by

$$
\begin{aligned}
|\Psi(t)\rangle & =e^{i \theta t-i \Gamma_{I}(t)}\left[a|00\rangle \otimes|\alpha(t)\rangle_{E}+d|11\rangle \otimes|-\alpha(t)\rangle_{E}\right. \\
& \left.+e^{-i 2 \theta t+i \Gamma_{I}(t)}(b|01\rangle+c|10\rangle) \otimes|0\rangle_{E}\right],
\end{aligned}
$$

where the phase $\theta \equiv I_{\text {eff }} / \hbar=2 \lambda^{2} / \omega$ is due to the indirect coupling between the two qubits, the phase $\Gamma_{I}(t) \equiv$ 
$\left(\frac{2 \lambda}{\omega}\right)^{2} \sin \omega t$ is due to the action of a displacement operator on the coherent state, and $|\alpha(t)\rangle_{E}$ is a coherent state with $\alpha(t)=\frac{2 \lambda}{\omega}\left(e^{-i \omega t}-1\right)$. Notice that the subspace spanned by $\{|01\rangle,|10\rangle\}$ forms a decoherence free subspace 11]. In general, Eq. (3) shows entanglement among the two qubits and the harmonic oscillator, depending on the parameters: $\omega$ and $\lambda$.

For $\omega \gg \lambda,|\alpha(t)\rangle$ is very close to $|0\rangle$. This means the overlap $\langle 0 \mid \alpha(t)\rangle=\exp \left[-\Gamma_{R}(t)\right] \approx 1$ where $\Gamma_{R}(t) \equiv$ $\left(\frac{2 \lambda}{\omega}\right)^{2}(1-\cos \omega t)$ goes to 0 . Also the phase $\Gamma_{I}(t)$ becomes zero. It is interesting that these two phases, $\Gamma_{R}(t)$ and $\Gamma_{I}(t)$ can also be calculated from the correlation function 2, 8, 10

$$
{ }_{E}\left\langle 0\left|e^{\Phi(t)} e^{\Phi^{\dagger}(0)}\right| 0\right\rangle_{E} \equiv e^{-\Gamma(t)},
$$

where $\Phi \equiv-\frac{2 \lambda}{\omega}\left(a^{\dagger}-a\right)$ and $\Phi(t)=e^{i \omega t a^{\dagger} a} \Phi e^{-i \omega t a^{\dagger} a}$. It is straightforward to obtain $\Gamma(t)$ given by

$$
\begin{aligned}
\Gamma(t) & =\Gamma_{R}(t)+i \Gamma_{I}(t) \\
& =\left(\frac{2 \lambda}{\omega}\right)^{2}[(1-\cos \omega t)-i \sin \omega t] .
\end{aligned}
$$

The roles of the real and imaginary parts of $\Gamma(t)$ are explained below. Then, in the limit of $\omega \gg \lambda$ Eq. (3) can be written as a product state of the two qubits and the harmonic oscillator

$$
|\Psi(t)\rangle \approx\left[a|00\rangle+d|11\rangle+e^{-i 2 \theta t}(b|01\rangle+c|10\rangle)\right] \otimes|0\rangle_{E} .
$$

The harmonic oscillator remains isolated always from the system, but it induces the indirect interaction between the two qubits and thus entanglement between them [5]. For a pure two qubits, concurrence, an entanglement measure, reads $C_{\text {ideal }}=2\left|a d-e^{i 4 \theta t} b c\right|[12$. Here the subscript, ideal, stands for the case of the qubits in a pure state. If $a=b=c=d=1 / 2$, we have $C_{\text {ideal }}=|\sin 2 \theta t|$ as shown in Fig. 2

The above analysis shows that the common harmonic oscillator with the high frequency, compared with the coupling constant to qubits, induces an inter-qubit coupling and can be used as a quantum data bus. However, the induced qubit-qubit coupling $I_{\text {eff }}$ is very small, so that the operation time of two-qubit gates is much longer than that of single-qubit gates. Notice that some superconducting qubits use this kind of inter-qubit coupling scheme 2]. As shown below, we find that a much faster two-qubit gate operation is possible and the maximally entangled state of the two qubits is obtainable even without the restriction of $\omega \gg \lambda$.

The harmonic oscillator plays two competing roles: (i) making two qubits entangled by inducing the two-qubit coupling $I_{\text {eff }}$, (ii) having two qubits decoherent through entanglement with them, i.e. $\Gamma_{R}(t)$. The role of the imaginary part $\Gamma_{I}(t)$ is different from that of the real part $\Gamma_{R}(t)$. While $\Gamma_{R}(t)$ makes the two qubit decoherent, i.e. decaying the off-diagonal elements, $\Gamma_{I}(t)$ causes the twoqubit coupling to fluctuate as shown in Fig. 2(b). These could be uncovered by examining the reduced density matrix of the two qubits

$$
\rho(t)=\frac{1}{4}\left[\begin{array}{cccc}
1 & e^{i 2 \theta t-\Gamma^{*}(t)} & e^{i 2 \theta t-\Gamma^{*}(t)} & e^{-4 \Gamma_{R}(t)} \\
e^{-i 2 \theta t-\Gamma(t)} & 1 & 1 & e^{-i 2 \theta t-\Gamma(t)} \\
e^{-i 2 \theta t-\Gamma(t)} & 1 & 1 & e^{-i 2 \theta t-\Gamma(t)} \\
e^{-4 \Gamma_{R}(t)} & e^{i 2 \theta t-\Gamma^{*}(t)} & e^{i 2 \theta t-\Gamma^{*}(t)} & 1
\end{array}\right],
$$

which is obtained by partial tracing over the environment, $\rho(t)=\operatorname{Tr}_{E}\{|\Psi(t)\rangle\langle\Psi(t)|\}$. Entanglement of the two qubits in a mixed state is measured by the concurrence $C=\max \left\{r_{1}-r_{2}-r_{3}-r_{4}, 0\right\}$ where $r_{i}^{2}$ are eigenvalues of $\rho \cdot \sigma_{1 y} \otimes \sigma_{2 y} \cdot \rho^{*} \cdot \sigma_{1 y} \otimes \sigma_{2 y}[12]$. Because the total state is in a pure state, entanglement of the two qubits and the harmonic oscillator is quantified by the von Neumann entropy of the two qubits $S=-\operatorname{Tr}(\rho \log \rho)$.
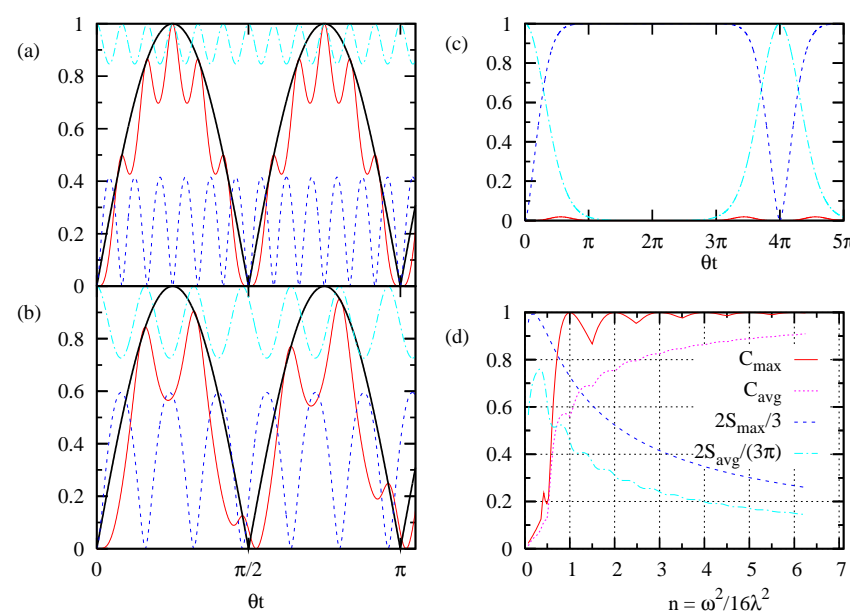

FIG. 2: (color online). Concurrence $C$ (red solid), $C_{\text {ideal }}$ (black thick solid), entropy $2 S / 3$ (blue dashed), and the overlap $\exp \left[-\Gamma_{R}(t)\right]$ (cyan dashed-dotted) as a function of time for $\omega / \lambda=4 \sqrt{3}$ (a), 5 (b), and 1 (c). (d) Maximum concurrence $C_{\max }$, maximum entropy $S_{\max }$, average concurrence $C_{\text {avg }}$, and average entropy $S_{\text {avg }}$ as a function of $n=(\omega / 4 \lambda)^{2}$ for $0 \leq \theta t<\pi / 2$.

As $\omega / \lambda$ becomes large, $C$ goes to $C_{\text {ideal }}$ and $S$ to 0 as depicted in Fig. 2. This is explained by means of the Born-Oppenheimer approximation that is based on the assumption of the weak coupling between the two qubits and the environment. The frequency $\omega$ of the harmonic oscillator is larger than that of the two qubits, $\theta$. The harmonic oscillator oscillates very fast in comparison with the two qubits. So the two qubits feel the harmonic oscillator stays in the same state. However, the condition of $\omega \gg \lambda$ is not a unique way to make the two qubits entangled maximally. Surprisingly, we find the maximum entanglement of the two qubits, $C=1$, at $\theta t=\pi / 4$ under the condition that $\omega / \lambda=4 \sqrt{n}$ with $n=1,2, \ldots$ as shown in Figs. 22 a) and 2(d). This is due to the fact that the frequency $\theta$ for entangling the two qubits is commensurate with the frequency $\omega$ of the harmonic oscillator. 
If the condition of $\omega / \lambda=4 \sqrt{n}, n=1,2, \ldots$ is not met, then the concurrence $C$ does not reach 1 at $\theta t=\pi / 4$ and 0 at $\theta t=\pi / 2$ due to $\Gamma(t)$ as shown in Fig. 2(b). The oscillation period of $C$ (red solid line) does not coincide with that of the ideal case $C_{\text {ideal }}$ (thick black line). This implies that the two qubit coupling fluctuates due to $\Gamma_{I}(t)$. For $\omega / \lambda<1$, our numerical study shows that the two qubits could not be entangled. Fig. 2(c) shows the case of $\omega=\lambda$.

Fig. 2(d) shows two competing roles of the harmonic oscillator. Let us define the average concurrence $C_{\text {avg }}$ for a period $\tau \equiv \pi / 2 \theta$ by $C_{\text {avg }} \equiv \frac{1}{\tau} \int_{0}^{\tau} C(t) d t$. For the first period $0 \leq \theta t<\pi / 2$, the maximum concurrence $C_{\max }$ reaches the peak at positive integers $n$ and the average concurrence $C_{\text {avg }}$ increases as $\omega / \lambda$ increases. In contrast, the maximum entropy $S_{\max }$ and the average entropy $S_{\text {avg }} \equiv \frac{1}{\tau} \int_{0}^{\tau} S(t) d t$ decrease. Due to the commensuration of two frequencies $\omega$ and $\theta, S_{\text {avg }}$ and $C_{\text {avg }}$ show the behavior of the stair case.

Two-spin boson model. - In the single mode case, the dynamics is reversible. If the single harmonic oscillator is replaced by the bosonic bath, the dynamics of qubits becomes irreversible in the sense that a pure initial qubit state becomes mixed and never returns to a pure state. The total Hamiltonian of the two-spin boson model is given by

$$
H=\sum_{j=1}^{N} \hbar \omega_{j} a_{j}^{\dagger} a_{j}+\left(\sigma_{1 z}+\sigma_{2 z}\right) \sum_{j=1}^{N} \hbar \lambda_{j}\left(a_{j}^{\dagger}+a_{j}\right),
$$

where the first term describes the bosonic bath and the second the interaction between the two qubits and the bosonic bath (environment). Here it is assumed that $N$ goes to infinity. The Hamiltonian of two qubits $H_{q}=$ $\sum_{i=1,2}\left(B_{i x} \sigma_{i x}+B_{i z} \sigma_{i z}\right)$ is set to be zero by turning off an external magnetic field. Also it is assumed that no direct two qubit coupling is available.

The environment is characterized by the spectral density function $J(\omega)=\sum_{j} \lambda_{j}^{2} \delta\left(\omega-\omega_{j}\right)$ [8, [9] . As shown in the inset of Fig. 3. (a), let us consider an Ohmic environment with a gap $\omega_{0}$ and an exponential cutoff function of the cutoff frequency $\omega_{c}$

$$
J(\omega)=\alpha\left(\omega-\omega_{0}\right) e^{-\left(\omega-\omega_{0}\right) / \omega_{c}} \theta\left(\omega-\omega_{0}\right),
$$

where $\alpha$ is the coupling parameter and $\theta(x)$ a step function.

Along the same lines of the single mode case, the canonical transformation

$$
e^{X}=\exp \left[\left(\sigma_{z 1}+\sigma_{z 2}\right) \sum_{j} \frac{\lambda_{j}}{\omega_{j}}\left(a_{j}^{\dagger}-a_{j}\right)\right]
$$

eliminates the coupling between the two qubits and the common environment in the Hamiltonian (8) and induces the indirect coupling between the two qubits. The transformed Hamiltonian becomes

$$
\widetilde{H}=\sum_{j} \hbar \omega_{j} a_{j}^{\dagger} a_{j}-I_{\mathrm{eff}} \sigma_{1 z} \sigma_{2 z}-I_{\mathrm{eff}}
$$
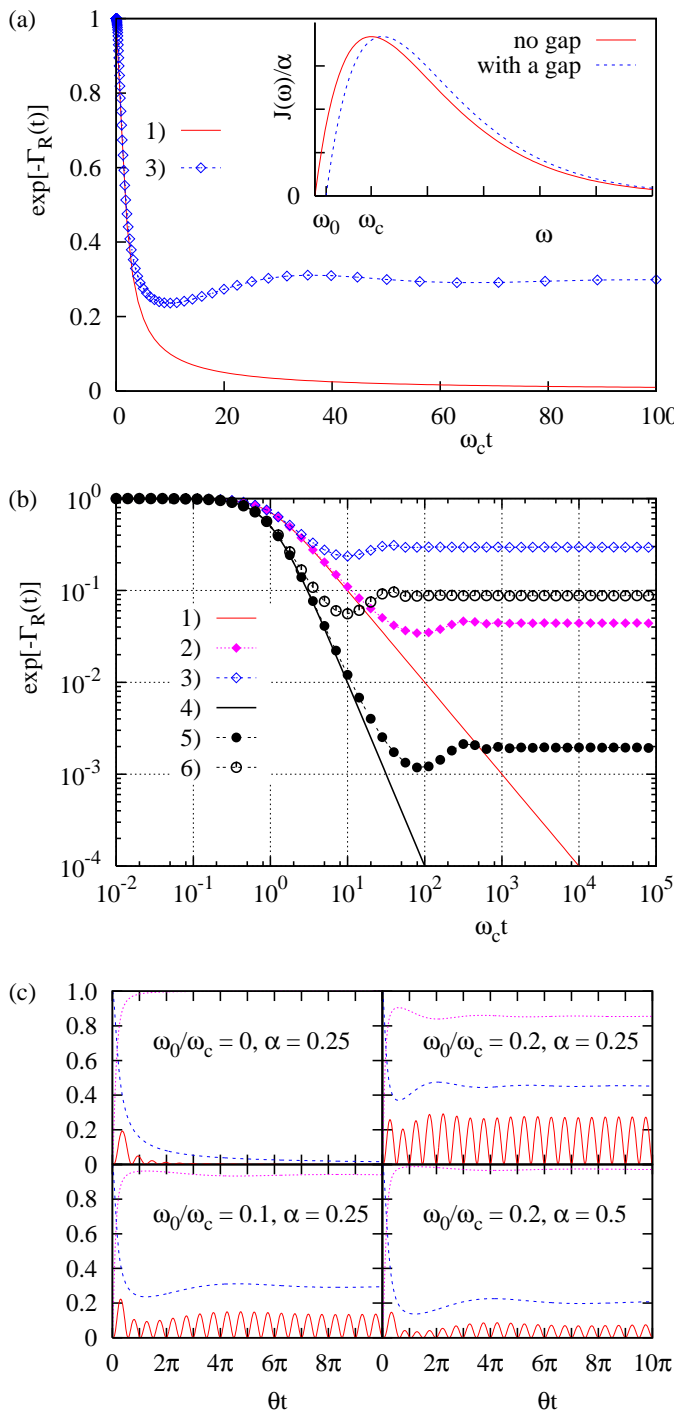

FIG. 3: (color online). (a) $\exp \left[-\Gamma_{R}(t)\right]$ as a function of $\omega_{c} t$ and (b) its $\log$ scale plot. Legends 1$\left.), \ldots, 6\right)$ refer to $\left(\omega_{0} / \omega_{c}, \alpha\right)=(0,0.25),(0.01,0.25),(0.1,0.25),(0,0.5)$, $(0.01,0.5),(0.1,0.5)$, respectively. Inset of $(\mathrm{a})$ : the spectral density function $J(\omega)$ with a gap $\omega_{0}$. (c) Concurrence $C$ (solid), entropy $2 S / 3$ (dotted), and $\exp \left[-\Gamma_{R}(t)\right]$ (dashed) as a function of $\theta t$.

where the induced coupling is given by

$$
I_{\mathrm{eff}} \equiv \hbar \sum_{j} \frac{2 \lambda_{j}^{2}}{\omega_{j}}=2 \hbar \int_{0}^{\infty} \frac{J(\omega)}{\omega} d \omega .
$$

For the Ohmic environment without a gap, $\omega_{0}=0$, we have $I_{\text {eff }}=2 \hbar \alpha \omega_{c}$.

Let us investigate the dynamics of entanglement. For an initial state $|\Psi(0)\rangle=|\psi(0)\rangle \otimes|\mathbf{0}\rangle_{E}$ where $|\mathbf{0}\rangle_{E}=$ $\left|0_{1}, 0_{2}, \ldots, 0_{N}\right\rangle$ is the ground state of $N$ harmonic oscil- 
lators, the state at time $t$ is given by

$$
\begin{aligned}
|\Psi(t)\rangle & =e^{i \theta t-i \Gamma_{I}(t)}\left[a|00\rangle \otimes|\boldsymbol{\alpha}(t)\rangle_{E}+d|11\rangle \otimes|-\boldsymbol{\alpha}(t)\rangle_{E}\right. \\
& \left.+e^{-i 2 \theta t+i \Gamma_{I}(t)}(b|01\rangle+c|10\rangle) \otimes|\mathbf{0}\rangle_{E}\right]
\end{aligned}
$$

where $|\boldsymbol{\alpha}(t)\rangle_{E} \equiv\left|\left\{\frac{\lambda_{i}}{\omega_{i}}\left(e^{-i \omega_{i} t}-1\right)\right\}\right\rangle$ is the collection of the coherent states. The imaginary part of $\Gamma(t)$ becomes

$$
\begin{aligned}
\Gamma_{I}(t) & =\sum_{j}\left(\frac{2 \lambda_{j}}{\omega_{j}}\right)^{2} \sin \omega_{j} t \\
& =4 \int_{0}^{\infty} \frac{J(\omega)}{\omega^{2}} \sin (\omega t) d \omega .
\end{aligned}
$$

Also we obtain the overlap ${ }_{E}\langle\mathbf{0} \mid \boldsymbol{\alpha}(t)\rangle_{E} \equiv \exp \left[-\Gamma_{R}(t)\right]$ where

$$
\Gamma_{R}(t)=4 \int_{0}^{\infty} \frac{J(\omega)}{\omega^{2}}(1-\cos \omega t) d \omega
$$

From Eq. (13), the density matrix of the two qubits becomes the same form of Eq. (7). For the Ohmic spectral density (9) without a gap, $\omega_{0}=0$, we have [8, 13]

$$
\begin{aligned}
\Gamma_{R}(t) & =2 \alpha \ln \left[1+\left(\omega_{c} t\right)^{2}\right], \\
\Gamma_{I}(t) & =4 \alpha \tan ^{-1}\left(\omega_{c} t\right) .
\end{aligned}
$$

The slopes of solid lines in Fig. 3(b) are $4 \alpha$. In this case $\exp \left[-\Gamma_{R}(t)\right]$ goes to zero as time goes on, but never returns to 1 in contrast to the single-mode case. Then the density matrix of the two qubits eventually becomes a mixed state with the maximum entropy $S=2 / 3$. For the environment without a gap, the two qubits become entangled and disentangled only for the transient times not in the "steady state" as shown in Fig. 3(c). Here the term "steady state" is used in the sense that in the steady state the entropy $S(t)$ of the two qubits does not change in time [14]. In this case, there is no further information flow from the two qubit to the environment.

It is clear that in the steady state the two qubits could be entangled and disentangled only if $\exp \left[-\Gamma_{R}(t)\right]$ remains finite. How is it possible? We find that the small gap $\omega_{0} \ll \omega_{c}$ of the Ohmic environment makes $\exp \left[-\Gamma_{R}(t)\right]$ finite in the steady state and the two qubits entangled. Fig. 3 shows that for the Ohmic environment with a gap the decoherence term $\exp \left[-\Gamma_{R}(t)\right]$ does not vanish in the steady state. The role of the gap is to prevent the complete drain of information from the qubits to the bath. We would like to mention a similar phenomenon, that is, the inhibition of the spontaneous emission of an atom in photonic-band-gap materials [15]. Notice that the gap of the spectral density function causes the phenomenon called false decoherence [16]. It would be interesting to derive a master equation of the two qubits in the Lindblad form when the spectral density of the environment has a gap.
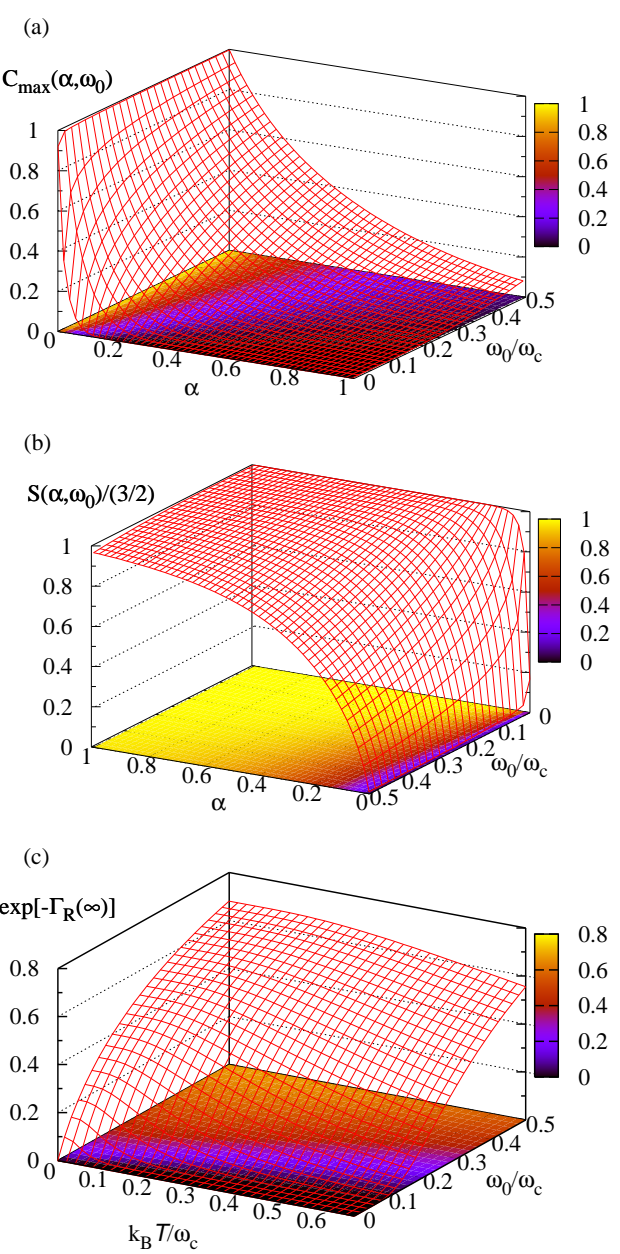

FIG. 4: (color online). (a) Maximum concurrence of the two qubits (b) entropy of two qubits at equilibrium as a function of $\alpha$ and $\omega_{0} / \omega_{c}$. (c) $\exp \left[-\Gamma_{R}(\infty)\right]$ as a function of temperature $T$ and $\omega_{0} / \omega_{c}$ for $\alpha=0.25$.

Figs. 4(a) and 4(b) show the maximum concurrence $C_{\max }$ and the entropy $S$ in the steady state at zero temperature. $C_{\max }(S)$ decreases (increases) as $\alpha$ increases even for $\omega_{0} \neq 0$. The effect of the non zero temperature of the environment is shown in Fig. 4(c). For finite temperatures, we take the initial density matrix $W(0)$ of the total system given by

$$
W(0)=|\psi(0)\rangle\langle\psi(0)| \otimes \rho_{E}, \quad \rho_{E}=\frac{e^{-H_{E} / k_{B} T}}{Z_{E}},
$$

where the environment is in a thermal equilibrium state $\rho_{E}$ at temperature $T$. Here $H_{E}=\sum_{i} \hbar \omega_{i} a_{i}^{\dagger} a_{i}$ and $Z_{E}$ is the partition function of the environment. At finite temperatures, the decoherence factor is given by 2 , 8, 10]

$$
\Gamma_{R}(t)=4 \int_{0}^{\infty} \frac{J(\omega)}{\omega^{2}} \operatorname{coth}\left(\frac{\hbar \omega}{2 k_{B} T}\right)(1-\cos \omega t) d \omega
$$

which decreases the effect of the gap as shown in Fig. 4(c). However, the real part $\Gamma_{R}(t)$ is identical to Eq. (14). 
We would like to make some remarks. First, it is easy to obtain the induced interaction for many qubits interacting with a common environment. It has the form of pair-wise interactions $\widetilde{H} \propto I_{\text {eff }} \sum_{i j} \sigma_{i z} \sigma_{j z}$. In this paper no external magnetic fields are applied on qubits, i.e., $\mathbf{B}_{i}, i=1,2$. For $\mathbf{B}_{i} \neq 0$, one can also obtain the effective Hamiltonian using the flow equation renormalization group [17], which is a generalized canonical transformation.

Second, there is an interesting physical systems similar to the two-spin boson model. Ref. [4] considers two spins of the two quantum dots coupled with a 2-dimensional electron gas. In this case the induced interaction is nothing but the Ruderman-Kittel-Kasuya-Yosida (RKKY) interaction. However, the spin on a quantum dot becomes entangled with a 2-dimensional electron gas [18]. Also the density of states of an electron gas has no gap. According to our theory in this paper, although the two spins are coupled via the RKKY interaction, their state is not pure but mixed. A further study should be done in order to know whether the RKKY interaction could be used for a two-qubit coupling scheme in solid state quantum computers [4].

In conclusion, we have studied the two qubits interacting with a common environment with a gap, described by a two-spin-boson model. For the environment of a single harmonic oscillator, if the induced two-qubit coupling strength is commensurate with the frequency of the harmonic oscillator, the two qubits could become maximally entangled. Also we discussed the roles of the real and imaginary parts of $\Gamma(t)$. In the case of the two-spin boson model with the spectral density function of the gap $\omega_{0}$, at the steady state the two qubits become entangled and disentangled due to the indirect interaction between qubits induced by the environment. Our result shows explicitly that two qubits can be entangled due to the environment with a gap while the environment itself is not entangled with qubits.

\section{Acknowledgments}

We would like to thank Howard Lee and Daniel Braun for helpful comments. This research was supported by a Grant(TRQCQ) from the Ministry of Science and Technology of Korea and by Korea Research Foundation Grant KRF-2004-041-C0089. This work was also partially supported by Korea Research Foundation Grant KRF-2002-070-C00029.
[1] M. A. Nielsen and I. L. Chuang, Quantum Computation and Quantum Information (Cambridge University Press, Cambridge, 2000).

[2] Y. Makhlin et al., Rev. Mod. Phys. 73, 357 (2001).

[3] S.-B. Zheng and G.-C. Guo, Phys. Rev. Lett. 85, 2392 (2000); A. Blais et al., Phys. Rev. A 69, 062320(2004).

[4] N. J. Craig et al., Science 304, 565 (2004).

[5] T. S. Cubitt, et al., Phys. Rev. Lett. 91, 037902 (2003).

[6] D. Braun, Phys. Rev. Lett. 89, 277901 (2002).

[7] F. Benatti, et al., Phys. Rev. Lett. 91, 070402 (2003)

[8] A. J. Leggett, et al., Rev. Mod. Phys. 59, 1 (1987); ibid. 67, 725 (1995).

[9] U. Weiss, Quantum Dissipative Systems (World Scientific,Singapore,1998).

[10] G. D. Mahan, Many-Particle Physics (Plenum Press, New York, 1990).

[11] L. M. Duan and G. C. Guo, Phys. Rev. Lett. 79, 1953 (1997); D. A. Lidar et al., ibid. 81, 2594 (1998).

[12] S. Hill and W. K. Wootters, Phys. Rev. Lett. 78, 5022
(1997); W. K. Wootters, ibid. 80, 2245 (1998).

[13] I. S. Gradshteyn and I. M. Ryzhik, Table of Integrals, Series, and Products (Academic Press, New York, 1979) p. 489.

[14] For more details of irreversibility and entropy production, see H.-P. Breuer and F. Petruccione, The Theory of Open Quantum Systems (Oxford University Press, New York, 2002) p. 128.

[15] S. John and T. Quang, Phys. Rev. A 50, 1764 (1994); E. Yablonovitch, Phys. Rev. Lett. 582059 (1987).

[16] A. J. Leggett in Quantum Computing and Quantum Bits in Mesoscopic Systems edited by A. J. Leggett, B. Ruggiero, and P. Silvertrini (Kluwer Academic/Plenum Publishers, New York, 2004).

[17] S. Oh and J. Kim, Conference proceedings of the 2004 ERATO Conference on Quantum Information Science, Tokyo (2004).

[18] S. Oh and J. Kim, Phys. Rev. B 73, 152407 (2006). 